\title{
Conduction in fully ionized liquid metals*
}

\author{
D. J. Stevenson \\ Laboratory of Atomic and Solid State Physics, Cornell University, Ithaca, New York 14850
}

N. W. Ashcroft ${ }^{\dagger}$

Institut für Festkörperforschung, Kernforschungsanlage, Jülich, Germany

(Received 10 September 1973)

\begin{abstract}
Electron transport is considered in high-density fully ionized liquid metals. Ionic structure is described in terms of hard-sphere-correlation functions and the scattering is determined from self-consistently screened point ions. Applications to the physical properties of the deep interior of Jupiter are briefly considered.
\end{abstract}

\section{INTRODUCTION}

We are concerned here with the problem of calculating the resistivity of dense conducting fluids consisting solely of massive point ions and a neutralizing gas of interacting electrons. Several systems of physical and astrophysical interest are included in a calculation assuming the following: (i) The density of the system is such that the electrons can be treated nonrelativistically. If $n_{e}$ is the electron density, this restriction can be stated as $r_{s} \gg 10^{-2}$, where $r_{s}$ is the usual linear measure of electron density

$$
n_{e}=\left(\frac{4}{3} \pi r_{s}^{3} a_{0}^{3}\right)^{-1} \text {. }
$$

(ii) The electron gas is degenerate. This is an implied restriction on the temperature, namely

$$
T \ll\left(6 \times 10^{5}\right) / r_{s}^{2} K .
$$

(iii) The first Born approximation is adequate for the calculation of electron scattering cross sections from the ionic system. This condition is satisfied for $r_{s} \leqslant 1 / Z$ (where $+\mathrm{Ze}$ is the charge on the point ion) and is discussed in detail in Appendix A. At lower densities (larger $r_{s}$ ), the validity of the results must be viewed with the caution normally attributed to low-order calculations in liquid metals.

(iv) The density-density-correlation function (static-structure factor) of the ionic system can be approximated reasonably well by regarding the ions as an assembly of impenetrable spheres. In the presence of an electron gas (and with due account for the effects of exchange, correlation, and the adiabatic response to ionic motion), the effective ion-ion interaction is characterized at short range by a steeply repulsive region, and at long range by a weak oscillatory tail. ${ }^{1}$ At sufficiently high density $\left(r_{s} \ll 1\right)$, the interaction between ions is expected to depart from the hard-core model and approach the simple screened interaction following from Thomas-Fermi theory (as used by Hubbard and Lampe ${ }^{2}$ ).

(v) The contribution to the resistivity from electron-electron collisions can be neglected. So long as the electron system is highly degenerate, this assumption is reasonable.

In Sec. II we outline the basis of the calculations for the conductivity, and in subsequent sections estimate the melting temperatures of these fully ionized systems. The extensions to alloys are also discussed, and insofar as they apply the results are considered in the context of the physical properties of the deep interior of Jupiter.

\section{CAlculation}

Within the adiabatic approximation we may write the resistivity of the dense ionized fluid of $N$ ions in volume $\Omega$ as

$$
\rho=m / n_{e} e^{2} \tau
$$

where the transport relaxation time $\tau$ is given by

$$
\frac{1}{\tau}=\left\langle\left\langle\frac{2 \pi}{\hbar} \frac{1}{\Omega} \int \frac{d \overrightarrow{\mathrm{k}}^{\prime}}{(2 \pi)^{3}}\left|V\left(\overrightarrow{\mathrm{k}}-\overrightarrow{\mathrm{k}}^{\prime}\right) \rho_{\overrightarrow{\mathrm{k}}-\overrightarrow{\mathrm{k}}}\right|^{2}\left(1-\cos \theta_{\overrightarrow{\mathrm{k}} \overrightarrow{\mathrm{k}}^{\prime}}\right) \delta\left(\epsilon_{F}-\epsilon_{k^{\prime}}\right)\right\rangle\right\rangle,
$$

with

$$
\epsilon_{k^{\prime}}=\hbar^{2} \boldsymbol{k}^{2} / 2 m, \quad \epsilon_{F}=\hbar^{2} k_{F}^{2} / 2 m,
$$

and

$$
k_{F}^{3}=3 \pi^{2} n_{e} .
$$

Equation (1) represents the ensemble average of the resistivity calculated in Born approximation for elastic scattering from each configuration of the ions described by the density components

$$
\rho_{\overrightarrow{\mathrm{k}}-\overrightarrow{\mathrm{k}}^{\prime}}=\sum_{i=1}^{N} e^{-i\left(\overrightarrow{\mathrm{k}}-\overrightarrow{\mathrm{k}}^{\prime}\right) \cdot \overrightarrow{\mathrm{R}}_{i}},
$$


where $\left\{\vec{R}_{i}\right\}$ is the instantaneous set of ionic positions. The matrix element of the (self-consistently screened) electron-ion scattering potential $V(r)$ is defined for plane-wave levels $|\vec{k}\rangle$ by

$\Omega\left\langle\overrightarrow{\mathrm{k}}|V| \overrightarrow{\mathrm{k}}^{\prime}\right\rangle=V\left(\overrightarrow{\mathrm{k}}-\overrightarrow{\mathrm{k}}^{\prime}\right)=\int_{\Omega} d \overrightarrow{\mathrm{r}} e^{-i\left(\overrightarrow{\mathrm{k}}-\overrightarrow{\mathrm{k}}^{\prime}\right) \cdot \overrightarrow{\mathrm{r}}} V(\overrightarrow{\mathrm{r}})$.

If the scattering is sufficiently weak (Appendix A), Eqs. (1) and (2) reduce, as originally shown by Ziman, ${ }^{3}$ to

$$
\rho=\frac{a_{0} \hbar}{e^{2}} \frac{4 \pi^{3} Z}{a_{0} k_{F}} \int_{0}^{1} d y y^{3} S(y) v^{2}(y),
$$

where $y=\left|\overrightarrow{\mathrm{k}}-\overrightarrow{\mathrm{k}}^{\prime}\right| / 2 k_{F}$, and $v(y)$ is the electronion interaction scaled to its long-wavelength limit $\left(\frac{2}{3} \epsilon_{F}\right)$. The quantity $a_{0} \hbar / e^{2}$ may be viewed as the atomic unit of resistivity and has the convenient practical value of $21.7 \mu \Omega \mathrm{cm} . S\left(\overrightarrow{\mathrm{k}}-\overrightarrow{\mathrm{k}}^{\prime}\right)$ is the liquid-structure factor defined by

$$
S(\overrightarrow{\mathrm{q}})=(1 / N)\left\langle\left\langle\rho_{q} \rho_{-q}\right\rangle\right\rangle-N \delta_{\overrightarrow{\mathrm{q}}, 0} .
$$

In the Percus-Yevick model ${ }^{4}$ (for hard spheres of diameter $\sigma), S(\overrightarrow{\mathrm{q}})$ is a function of the packing fraction $\eta$ given by

$$
\eta=\frac{1}{6} \pi n_{\text {ion }} \sigma^{3}, \quad n_{\text {ion }}=N / \Omega \text {. }
$$

For most classical fluids near their solidification points, ${ }^{4,5} \eta \approx 0.45$.

We are dealing with point ions and the accuracy with which $v(y)$ can be specified is limited only by the uncertainties in the dielectric function $\epsilon(y)$. In the neighborhood of $y \sim 1$ [the regime dominating the integrand of (5)], $\epsilon(y)$ is quite well known and we take the interpolation form suggested by Hubbard, ${ }^{6}$ so that

$$
v(y)=-0.166 r_{s} /\left[y^{2}+0.166 r_{s} F(y)\right],
$$

where

$$
\begin{aligned}
& F(y)=f(y) /\left[1-0.166 r_{s} f(y)\left(2 y^{2}+g\right)^{-1}\right], \\
& g=\left(1+0.0262 r_{s}\right)^{-1},
\end{aligned}
$$

and

$$
f(y)=\frac{1}{2}+\frac{1-y^{2}}{4 y} \ln \left|\frac{1+y}{1-y}\right| .
$$

In practice, the replacement of $F(y)$ in (8) by the Lindhard function $f(y)$ leads to the same resistivity (to within $2 \%$ ), but the exchange and correlation corrections contained in $F(y)$ are important in calculations of quantities involving $[(1 / \epsilon)-1]$, such as the effective pair interaction between ions.

Since $\left(r_{s} a_{0} k_{F}\right)^{3}=\frac{1}{4}(9 \pi)$, we may rewrite (5) [using (8)] as

$$
\begin{aligned}
\rho /\left(r_{s}^{3} Z\right)= & 38.4 \\
& \times \int_{0}^{1} d y y^{3} S(y)\left[y^{2}+0.166 r_{s} F(y)\right]^{-2} \mu \Omega \mathrm{cm} .
\end{aligned}
$$

The utility of this expression is that the righthand side is, for $r_{s} \lesssim 1$, a weak function of $r_{s}$ and hence density. Figure 1 demonstrates this clearly. It is worth noting that the charge $Z$ enters in the structure factor. ${ }^{7}$

To obtain the resistivity as a function of temperature, we require $T(\eta)$ at each density. This can

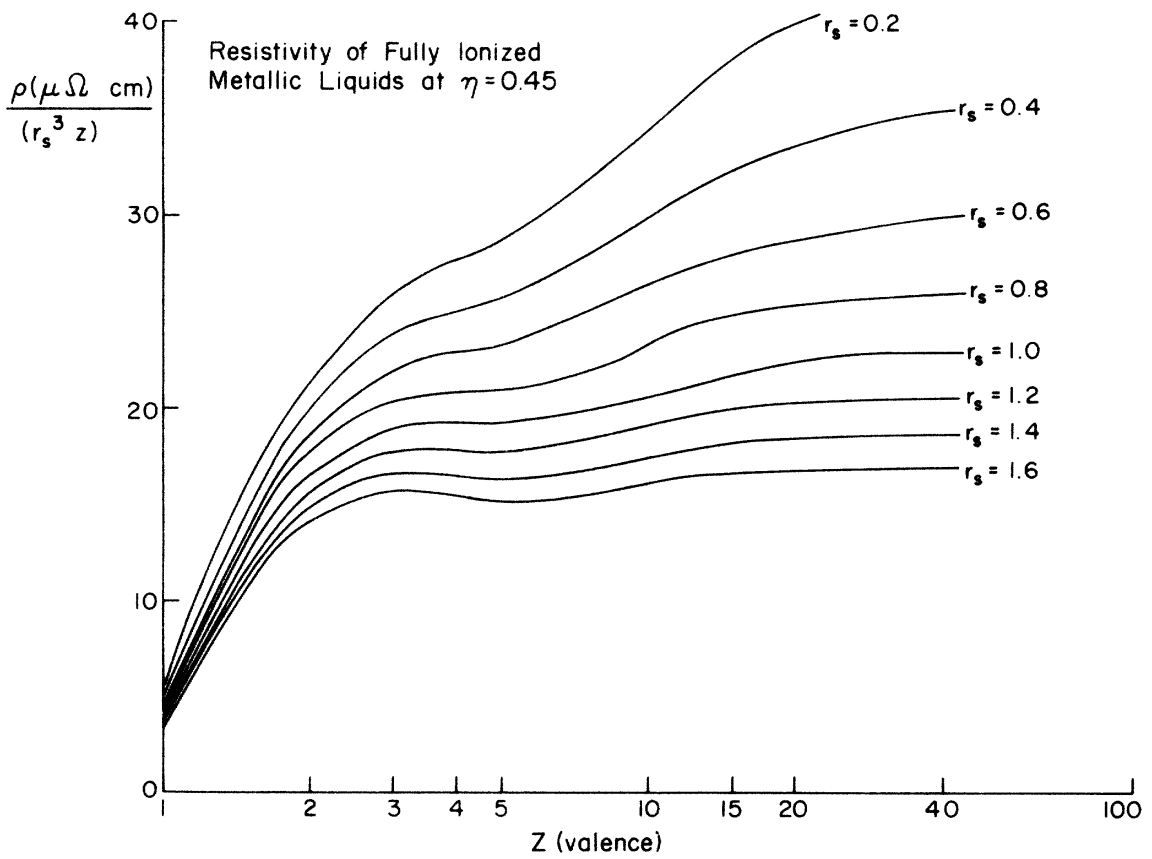

FIG. 1. Resistivity of fully-ionized liquids at $\eta=0.45$. 


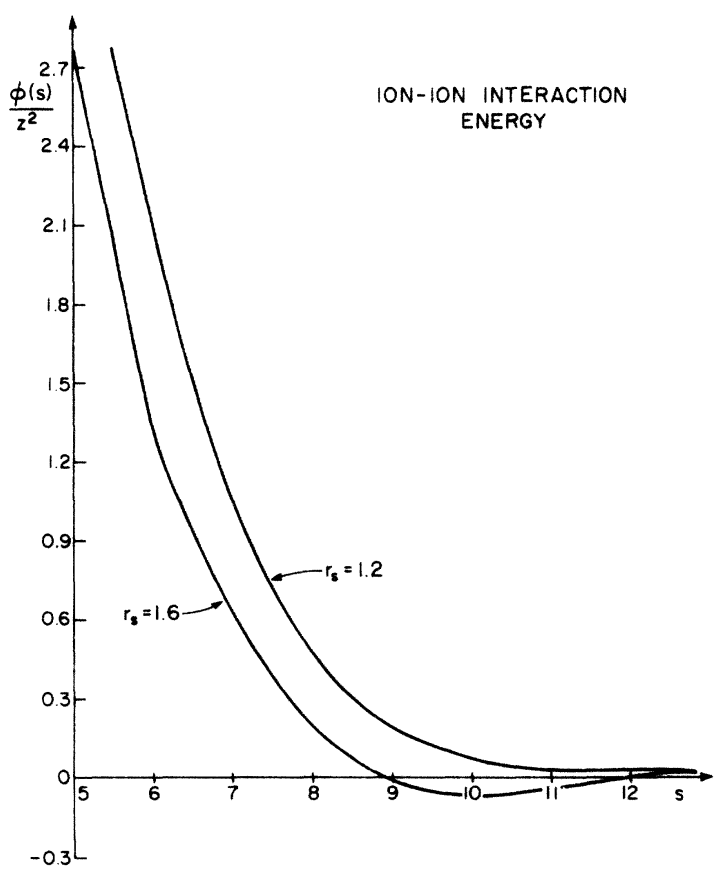

FIG. 2. Effective ion-ion interaction energy in units of $10^{-2} \epsilon_{F}$.

be obtained from a variational technique, ${ }^{8}$ but the method is laborious and for the present purposes it is sufficient to use the approximate technique suggested by Ashcroft and Langreth. ${ }^{1}$ We evaluate the pair interaction between point ions from

$$
\phi(s)=\left(0.166 r_{s}\right) \frac{8 Z^{2}}{s} \int_{0}^{\infty} \frac{x \sin s x}{x^{2}+0.166 r_{s} F(x)} d x,
$$

which gives the pair energy at separation $r(r=s /$ $2 k_{F}$ ) in units of $\epsilon_{F}$ (see Fig. 2). If $\phi_{\min }$ is the minimum value of $\phi(s)$, then the melting temperature $T_{N}$ can be estimated from the relation

$$
\phi\left(2 k_{F} \sigma\right)-\phi_{\min }=\frac{3}{2} k_{B} T_{M} / \epsilon_{F},
$$

provided $2 k_{F} \sigma$ is evaluated at $\eta=0.45$. It may be noted that this procedure gives $T_{M}$ in sodium to within $10 \%$. The same close agreement is not likely for fully ionized systems that have somewhat "softer" pair potentials (in reduced units) than that appropriate for sodium. ${ }^{1}$ To find $d \eta / d T$, we evaluate the slope of $\phi(s)$

$$
\frac{d \eta}{d T}=\left[9 \eta / 2 s\left(\frac{d \phi}{d s}\right) T_{F}\right]_{s=2(18 \pi Z \eta)^{1 / 3}},
$$

(where $T_{F}=\left(6 \times 10^{5}\right) / r_{s}^{2} \mathrm{~K}$ ) and in this way obtain $T_{M}$ (see Fig. 3) and the values of $T$ appropriate to $\eta<0.45$. An alternative method for obtaining $T_{M}$ exploits the Lindemann rule (see Appendix B), but the simpler approach outlined above is no less accurate and is, in fact, more fundamental.

The results of our calculation for fully ionized $\mathrm{H}, \mathrm{He}$, and $\mathrm{C}$ are found summarized in Figs. 4, 5 , and 6 , respectively. We choose as a vertical axis the quantity (resistivity $\times$ density), since, as noted above, this combination, near $T_{M}$, is weak-

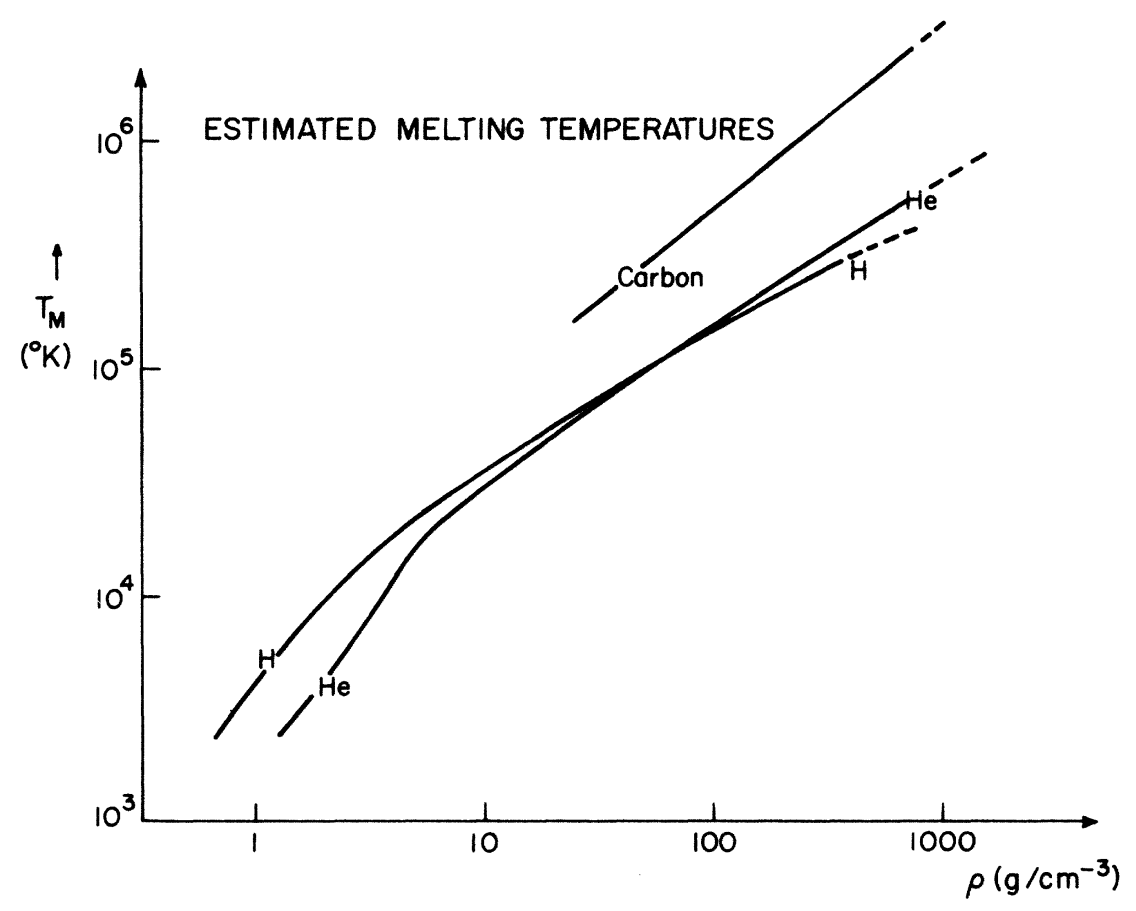

FIG. 3. Estimated melting temperatures. 


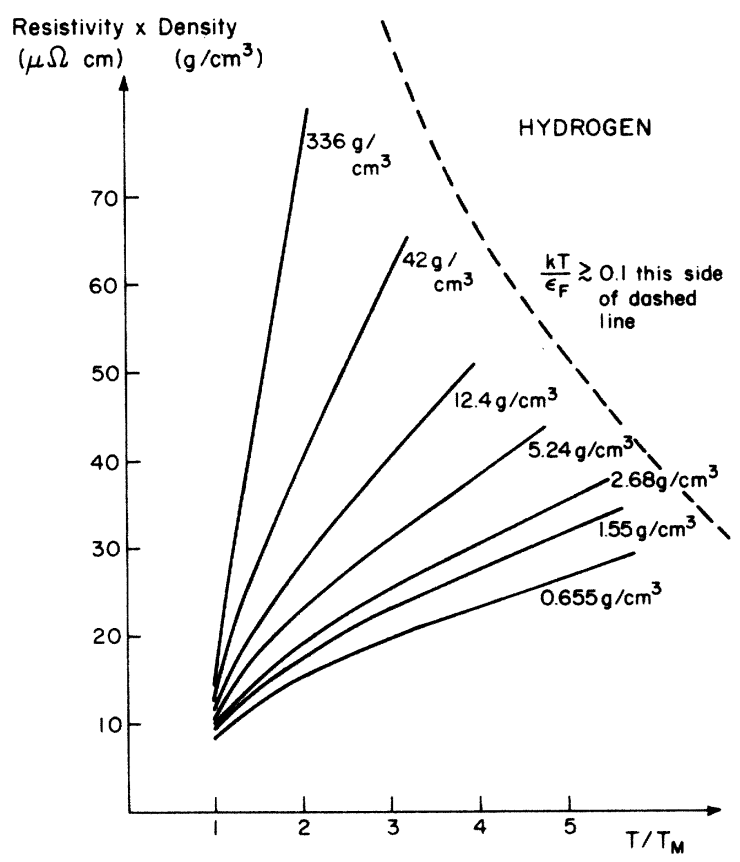

FIG. 4. Resistivity of hydrogen.

ly density dependent. It should be emphasized that if our estimates of $T_{\mu}$ are incorrect, the form of the curves presented will remain substantially correct. We should also point out that at densities for which the element carbon is likely to be fully pressure ionized, the hard-sphere approximation to the ion-ion interaction may already depart sub-

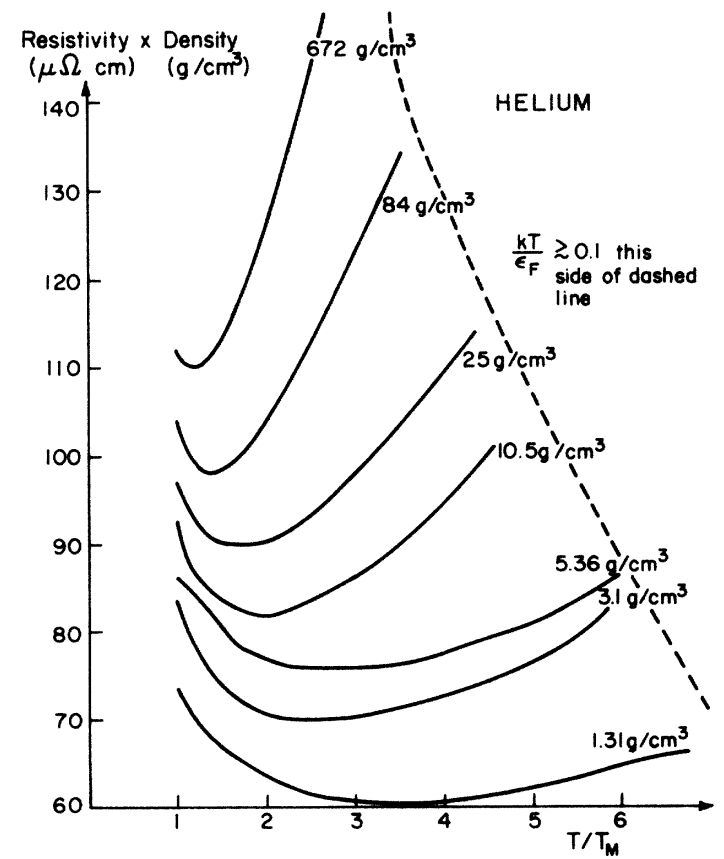

FIG. 5. Resistivity of helium. stantially from reality. ${ }^{9}$ Moreover, $k T_{M} / \epsilon_{F} \sim 0.05$, and this implies a significant nondegeneracy.

Figure 7 shows a comparison of our results with those of Hubbard and Lampe. ${ }^{2}$ The quantity compared is the conductive opacity ${ }^{10}$ as tabulated in Ref. 2. Our results are seen to be systematically lower, and the greatest difference occurs at low temperatures, where the crude approximation for $S(q)$ used in Ref. 2 is expected to be least accurate. We cannot, however, eliminate the possibility that the systematic discrepancy results from a disagreement in the temperature scale.

\section{EXTENSION TO ALLOYS}

The extension to binary alloys is straightforward in principle. ${ }^{1}$ The result equivalent to Eq. (5) can be written

$$
\begin{aligned}
\frac{\rho}{\left(r_{s}^{3} Z^{*}\right)}= & 38.4 \int_{0}^{1} \frac{y^{3} d y}{\left.y^{2}+0.166 r_{s} F(y)\right]^{2}} \\
& \times\left[x S_{22}(y)+2 x^{1 / 2}(1-x)^{1 / 2} S_{12}(y)\right. \\
& \left.+(1-x) S_{11}(y)\right] \mu \Omega \mathrm{cm},
\end{aligned}
$$

where $x$ is the fractional number of ions of species $2, Z^{*}$ is the number of electrons per ion, and $S_{22}$, $S_{12}, S_{11}$ are partial structure factors. ${ }^{11}$ These structure factors not only depend on

$$
\eta=\frac{\text { volume occupied by hard spheres }}{\text { total volume }}
$$

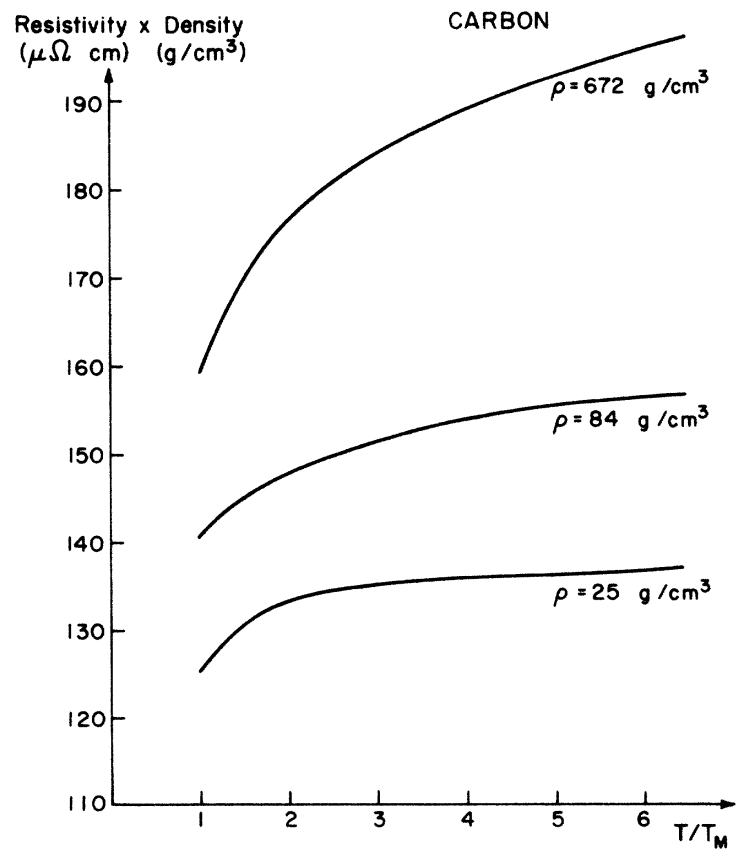

FIG. 6. Resistivity of carbon. 


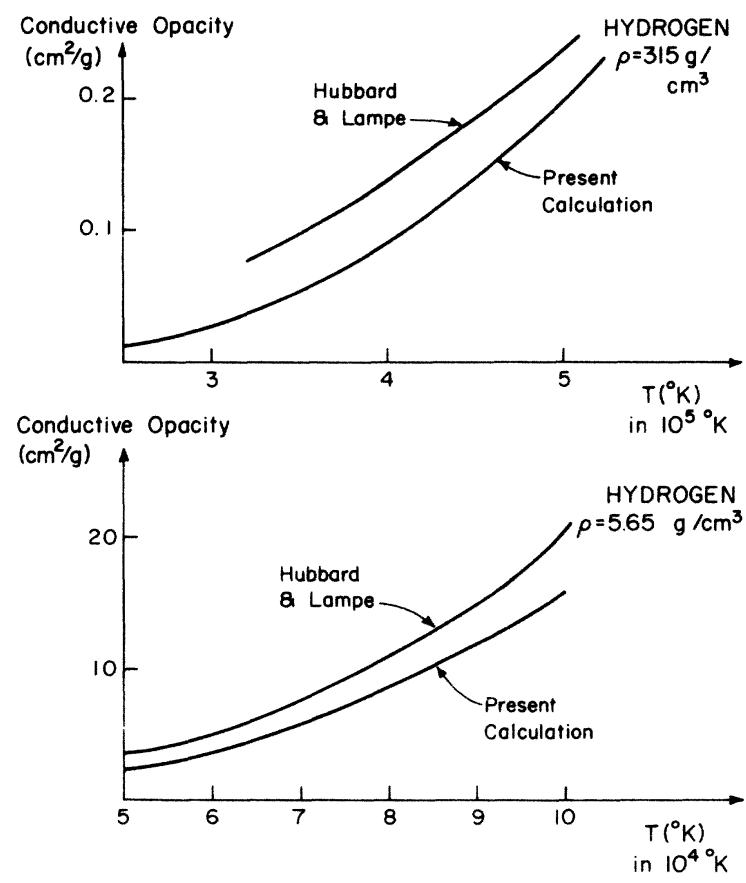

FIG. 7. Conductive opacity of hydrogen at two densities. A comparison of our results with those of Hubbard and Lampe (Ref. 2).

but also on

$$
\alpha=\sigma_{1} / \sigma_{2},
$$

where $\sigma_{1}$ and $\sigma_{2}$ are the hard-sphere diameters of components 1 and 2, respectively.

If $\alpha=1$, then Eq. (12) becomes identical to Eq.
(9), except, of course, that $Z^{*}$ is a function of $x$. In this special case, the results of Fig. 1 can be used to find the resistivity of any alloy ${ }^{12}$ at the melting point.

Equation (10) shows that if $\varphi\left(s_{0}\right)=0$ for the interaction between ions of species 1 , then $\varphi\left(s_{0}\right)=0$ for the ions of species 2. This suggests that $\alpha$ is near unity. However, the species with higher ionic charge is expected to have a "harder" core (for a given value of $r_{s}$ ). A detailed calculation ${ }^{13}$ suggests that $\alpha=0.75$ for a hydrogen-helium mixture; that is, the helium hard-sphere diameter is one-third larger than the hydrogen hard-sphere diameter. In Fig. 8, we show that this deviation from $\alpha=1$ does not dramatically change the resistivity, and accordingly a reasonable approximation sets all hard-sphere diameters equal.

There is, however, no simple extension of our method for obtaining $d \eta / d T$ to the alloy problem. For $Z>2$, the temperature dependence of the resistivity is sufficiently weak that it may be ignored in a first approximation (for $T_{M}<T \ll T_{F}$ ). For a hydrogen-helium alloy, a crude approximation simply interpolates between the temperature trends shown in Figs. 4 and 5 .

\section{SUMMARY AND APPLICATION}

In the limited temperature and density range appropriate to Eq. (5) and the hard-sphere model, we find somewhat lower resistivities than those previously obtained ${ }^{2}$ for fully ionized liquid metals. This is attributable to the use of a more accurate

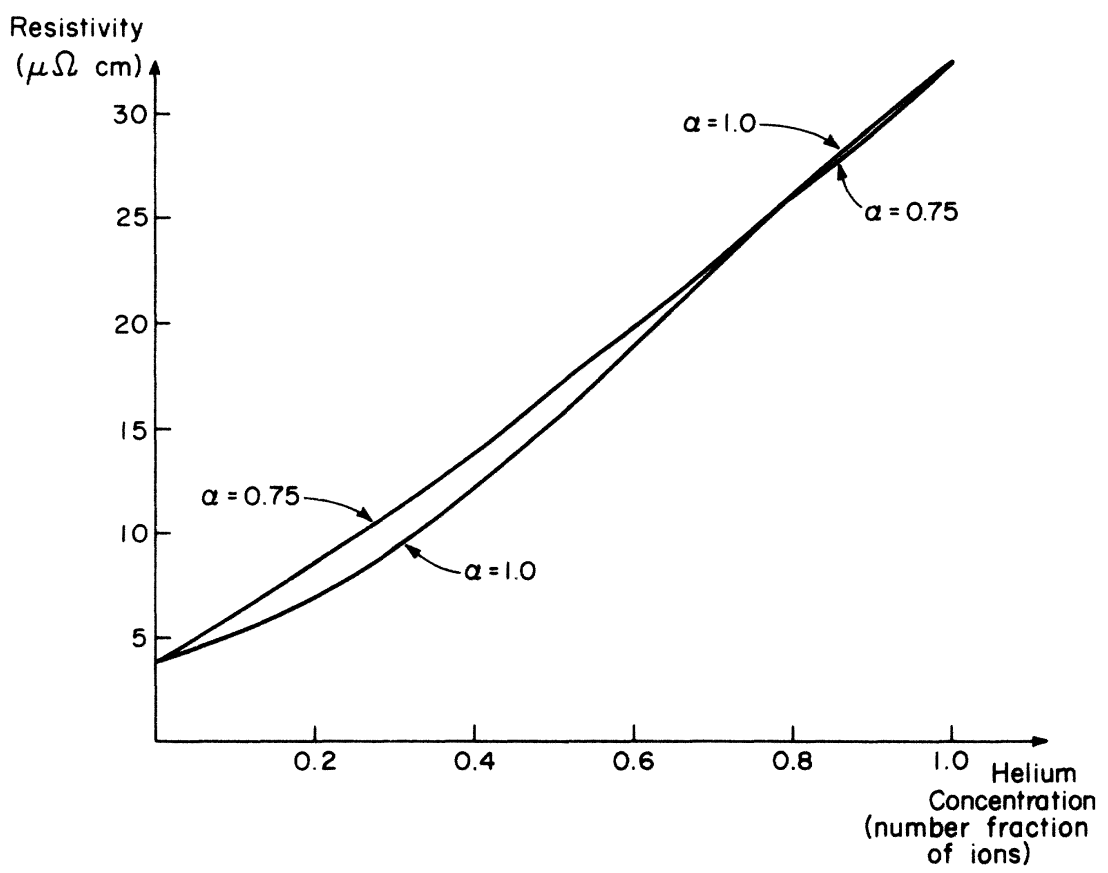

FIG. 8. Resistivity of an $\mathrm{H}-\mathrm{He}$ alloy at $r_{s}=1.0$ and $\eta=0.45$. The effect of diferent hard-sphere diameters is shown. 
electron-ion interaction and a more appropriate structure factor. A disadvantage of the present method is the need independently to estimate the temperature scale.

Systems for which the present calculations seem likely to apply include the interiors of the giant planets, in particular Jupiter. Most recent models of the Jovian interior postulate a central region of dense fluid. Its composition is predominantly metallic hydrogen, but is augmented by a small amount of helium (about $10 \%$ by number ${ }^{14,15}$ ). It is conceivable that the helium may not be completely ionized and if not, the electron-helium interaction may be more appropriate to that expected of neutral helium atoms. ${ }^{16}$ We find that although it is possible for the resistivity to be enhanced if the helium remains un-ionized, this enhancement is mainly a consequence of the small increase in the value of $r_{s}$, rather than any substantial change in the scattering cross section from that expected for fully ionized atoms.

If we choose the central temperature ${ }^{17}$ of Jupiter to be about $16000 \mathrm{~K}$, then we find that the resistivity of the fluid is expected to range from $4 \mu \Omega \mathrm{cm}$ at the center of Jupiter to about $8 \mu \Omega \mathrm{cm}$ at the boundary between metallic and molecular hydrogen. A conductivity characteristic of the deep interior of Jupiter is therefore

$$
\sigma \sim 2 \times 10^{17} \mathrm{esu},
$$

a result somewhat larger than most previous estimates. ${ }^{18}$

Jupiter is observed to have a strong magnetic field, and in seeking internal mechanisms for its origin it is first of interest to decide whether the field could be primordial. If it were, then the quantity of central importance is the decay time $T$ given in seconds by

$$
T \sim 4 \pi \sigma(L / c)^{2},
$$

where $c$ is the velocity of light and $L$ is a typical planetary dimension, which we take here as $5 \times 10^{9} \mathrm{~cm}$. The result

$$
T \sim 2 \times 10^{9} \text { years }
$$

may be seen to hinge not too seriously on the choice of $L$. Even if the value chosen is viewed as unreasonably large, the result for $T$ remains such that the possibility of primordial origin is difficult to discount. In complete contrast to this, it is interesting to record that the high value of $\sigma$ is likely to be favorable for a dynamo mechanism ${ }^{19}$ underlying the generation of the magnetic field.

Finally, a straightforward application of the Wiedemann-Franz relation yields thermal conductivities for the interior of Jupiter ranging from (in $\mathrm{erg} / \mathrm{cm} \mathrm{sec} \mathrm{K}$ ) $9 \times 10^{8}$ at the center to $1 \times 10^{8}$ at the metallic boundary. Now the observed internal heat flux is very high, ${ }^{20}$ but it is apparent that even conductivities of this magnitude are insufficient to maintain the measured flux unless we assume a much larger central temperature. ${ }^{14,15}$ In a situation such as this, the system is unstable against convection, and the planet would rapidly cool. It would seem to follow that all but a small core of Jupiter must be convective. The size of this convective region is an open question.

\section{ACKNOWLEDGMENTS}

One of the authors (N. W. A.) would like to thank Professor G. Eilenberger and his colleagues at the KFA, Jülich, for their kind hospitality during the period when part of this work was completed.

\section{APPENDIX A: VALIDITY OF THE BORN APPROXIMATION}

An elementary criterion for the validity of the Born approximation is that ${ }^{2}$

$$
Z e^{2} / \epsilon_{\boldsymbol{F}} \lesssim\left(\hbar^{2} / 2 m \epsilon_{\boldsymbol{F}}\right)^{1 / 2} .
$$

Here, the left-hand side is roughly the distance from the ion within which the interaction energy exceeds the Fermi energy. The right-hand side is of the order of the electron wavelength. It follows that

$$
\epsilon_{F} z 2 Z^{2} e^{2} /\left(\hbar^{2} / m e^{2}\right)=4 Z^{2} \mathrm{Ry},
$$

whence $r_{s} \lesssim 1 / Z$.

An alternative criterion is

$$
\sigma_{\text {Born }} / 4 \pi a^{2} \ll 1,
$$

where $4 \pi a^{2}$ is the "geometric" cross section. For a single ion

$$
\mathrm{O}_{\text {Born }}=\frac{1}{2 \pi k_{F}^{2}} \int_{0}^{2 k^{k}} k\left(\frac{m}{\hbar^{2}} V(k)\right)^{2} d k,
$$

where

$$
V(k)=\frac{4 \pi}{k} \int_{0}^{\infty} \sin k r V(r) r d r .
$$

We calculate $\sigma_{\text {Born }}$ approximately using ThomasFermi screening, i.e.,

$$
V(r)=\left(Z e^{2} / r\right) e^{-q_{s} r},
$$

so

$$
V(k)=\frac{4 \pi Z e^{2}}{k^{2}+q_{s}^{2}}=\frac{4 \pi Z e^{2}}{x^{2}+0.166 r_{s}} \frac{1}{\left(2 k_{F}\right)^{2}},
$$

where

$$
x=k / 2 k_{F} .
$$

Thus, 


$$
\sigma_{\text {Born }}=\frac{1}{2 \pi k_{F}^{2}}\left(\frac{4 \pi Z}{a_{0}}\right)^{2} \frac{1}{\left(2 k_{F}\right)^{2}} \int_{0}^{1} \frac{x d x}{\left(x^{2}+0.166 r_{s}\right)^{2}} .
$$

But,

$$
a \simeq 1 / q_{s}=0.64 r_{s}^{1 / 2} a_{0},
$$

and thus it follows that

$$
\sigma_{\text {Born }} / 4 \pi a^{2} \simeq\left(0.27 r_{s}^{2} Z^{2}\right) /\left(1+0.166 r_{s}\right) .
$$

Finally,

$$
\sigma_{\text {Born }} / 4 \pi a^{2} \ll 1 \text { implies } r_{s} \lesssim 1 / Z \text { (as before). }
$$

However, the Born cross section per ion in the condensed state is clearly different from that of a single isolated ion. We can calculate the "apparent" cross section, per ion, in the liquid by using the identity

$$
n \sigma_{a} v_{F} \tau \equiv 1,
$$

where $\tau$ is the "collision time" for an electron and $n$ is the ion number density.

Since $\rho=m / n_{\mathrm{el}} e^{2} \tau$, we have, from Eq. (5),

$$
\begin{aligned}
\sigma_{a} & =\frac{4 \pi^{3} Z^{2}}{k_{F}^{2}} \int_{0}^{1} y^{3} v^{2}(y) S(y) d y, \\
& =Z(\rho(\mu \Omega \mathrm{cm}) / 21.7)\left(r_{s} / 1.92\right) a_{0}^{2},
\end{aligned}
$$

whence
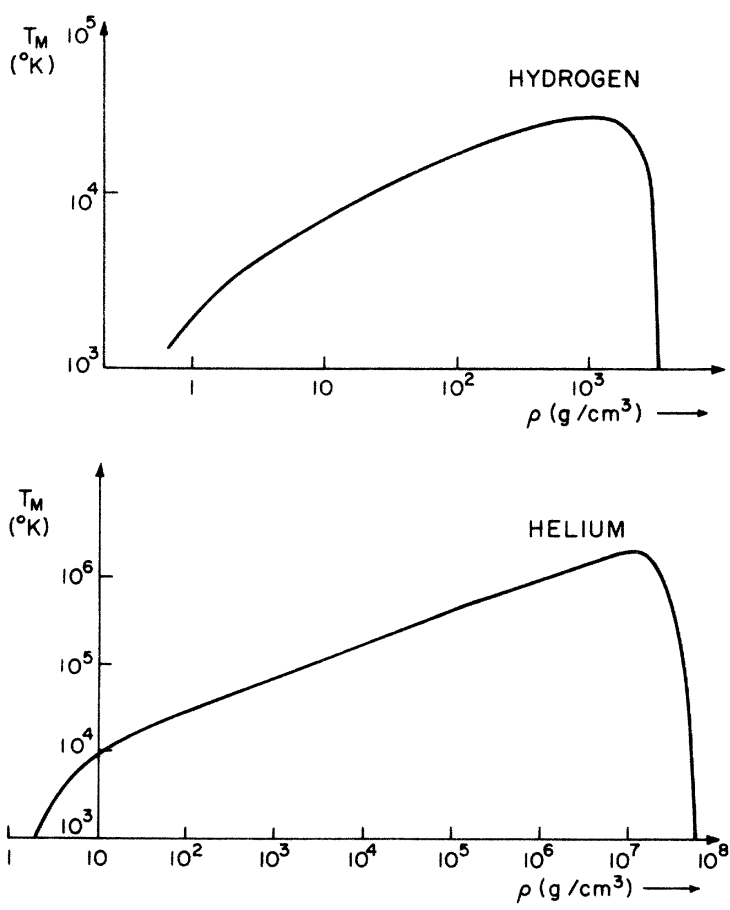

FIG. 9. The melting temperatures of metallic hydrogen and helium according to Lindemann's rule. $\sigma_{a} / 4 \pi a^{2} \simeq 0.1 Z(\rho(\mu \Omega \mathrm{cm}) / 21.7)$,

where $\rho$ is calculated from the first Born approximation. (Note that this formula is valid for any' simple liquid metal.)

For hydrogen at $r_{s}=1.6, \quad T=T_{M}$, we have $\sigma_{a} /$ $4 \pi a^{2} \simeq 0.06$, and for helium at $r_{s}=1.2, T=T_{k}$, we have $\sigma_{a} / 4 \pi a^{2} \simeq 0.25$.

This suggests (but does not prove) that the Born approximation may be much better satisfied in the condensed state than for a single ion. Thus, our criterion $r_{s} \lesssim 1 / Z$ may be too stringent. It is clear and expected, however, that the Born approximation is increasingly well satisfied as $r_{s}$ becomes smaller.

\section{APPENDIX B: MELTING CRITERION}

A commonly used criterion is Lindemann's rule. This can be written $\mathrm{as}^{21}$

$$
\gamma=\sum_{k \lambda} \frac{\hbar\left(n_{k \lambda}+\frac{1}{2}\right)}{\mathrm{Mn}_{\text {ion }} \omega_{k \lambda} R_{0}^{2}},
$$

where $\gamma$ is the mean-square amplitude of the ions just below the melting point and is found, almost universally, to be about $\frac{1}{16} . M$ is the ion mass, $\boldsymbol{R}_{0}$ the interatomic spacing, $\omega_{k \lambda}$ a phonon frequency of wave vector $k$ and polarization $\lambda$, and $n_{k \lambda}$ is the Bose-Einstein occupation factor.

For the high-density systems considered, Abrikosov $^{22}$ has shown that it is important to distinguish between the longitudinal and transverse modes, since the former are primarily determined by the bulk compressibility of the electron gas, whereas the latter are primarily determined by the Coulomb forces between ions.

We make a Debye approximation, but allow for the longitudinal and transverse "Debye" temperatures to be different. Using the method outlined by Trubitsyn, ${ }^{23}$ we obtain (in $\mathrm{K}$ )

$$
\begin{aligned}
& \Theta_{l} \simeq \frac{2500 Z^{1 / 6}}{A^{1 / 2}}\left(\frac{22.1}{r_{s}^{4}}-\frac{3.66}{r_{s}^{3}}-\frac{7.17 Z^{2 / 3}}{r_{s}^{3}}\right)^{1 / 2}, \\
& \Theta_{t} \simeq 8000\left(Z / \mathrm{Ar}_{s}^{3}\right)^{1 / 2} ;
\end{aligned}
$$

The correlation energy of the electron gas is small and can be ignored. Equation (B1) can then be written

$$
\begin{aligned}
& \frac{k_{B} \Theta_{l}}{M S_{l}^{2}}\left[1+4\left(\frac{T}{\Theta_{l}}\right)^{2} \int_{0}^{\Theta_{l} / T} \frac{x d x}{e^{x}-1}\right] \\
& \quad+\frac{2 k_{B} \Theta_{t}}{M S_{t}^{2}}\left[1+4\left(\frac{T}{\Theta_{t}}\right)^{2} \int_{0}^{\Theta_{t} / T} \frac{x d x}{e^{x}-1}\right] \simeq 0.47,
\end{aligned}
$$

where $S_{l}, S_{t}$ are the appropriate sound velocities. We anticipate $T_{M}<\Theta_{l}, \Theta_{t}$ and so approximate $\Theta_{l} / T$, 
$\Theta_{t} / T$ by $\infty$ in the integrals. It is easy to show that this is valid provided

$$
(\Theta / T) e^{-\Theta / r_{<} \frac{1}{6} \pi^{2}} \text { for } \Theta=\Theta_{l} \text { and } \Theta_{t},
$$

which is satisfied reasonably well for the cases studied. Equation (B2) can be written in numerical form, for low temperatures, as

$$
\frac{0.22}{A^{1 / 2} Z^{5 / 6}\left[\left(22.1 / r_{s}^{4}\right)-\left(3.66 / r_{s}^{3}\right)-\left(7.17 Z^{2 / 3} / r_{s}^{3}\right)\right]^{1 / 2} r_{s}^{2}}\left[1+\frac{2 \pi^{2}}{3}\left(\frac{T}{\Theta_{l}}\right)^{2}\right]+\frac{0.13}{A^{1 / 2} Z^{7 / 6} r_{s}^{1 / 2}}\left[1+\frac{2 \pi^{2}}{3}\left(\frac{T}{\Theta_{t}}\right)^{2}\right] \simeq 0.47
$$

and is solved to obtain $T_{M}$ (note that for $r_{s} \ll 1$, only the transverse modes are important in determining $T_{M}$ ). The results, shown in Fig. 9, give melting temperatures which differ by as much as a factor of 2 from those in Fig. 3. Similar results have been obtained by Pollack and Hansen. ${ }^{24}$ The problem with Lindemann's rule is that an error in $\gamma\left(=\frac{1}{16}\right.$ in the above calculation $)$ propagates alarmingly through to the final calculation of $T_{w}$, in the case $T_{M}<\Theta_{t}, \Theta_{l}$. Typically, a $10 \%$ error in $\gamma$ will give a $50 \%$ error in $T_{M}$. Moreover, our estimates of $\Theta_{t}, \Theta_{l}$ are only approximate. (Our formula for $\Theta_{l}$ is, however, in excellent agreement with the $\Theta_{D}$ calculated by Neece, Rogers, and Hoover. ${ }^{25}$ ) Note that at sufficiently high densities, the zero-point motion alone will cause the lattice to melt. Lindemann's rule gives an estimate of the value of $r_{s}^{1 / 2}$ at which $T_{M} \rightarrow 0$. Since density varies as $\left(r_{s}^{1 / 2}\right)^{-6}$, the density at which $T_{M} \rightarrow 0$ cannot be calculated to better than an order of magnitude using Lindemann's rule. (The pressure at which $T_{M} \rightarrow 0$ may be incorrect by almost two orders of magnitude.) As Abrikosov ${ }^{15}$ observes, only hydrogen and helium will melt at absolute zero and sufficiently high densities. This is because the densities required for heavier elements are such that the sizes of the nuclei become important.
*Supported in part by NASA under Contract No. NGR-33$010-188$, and by the National Science Foundation under Contract No. GH-36457.

$\dagger$ Permanent address: Laboratory of Atomic and Solid State Physics, Cornell University, Ithaca, N. Y. 14850.

${ }^{1}$ N. W. Ashcroft and D. C. Langreth, Phys. Rev. $\underline{159}$, 500 (1967).

${ }^{2}$ W. B. Hubbard and M. Lampe, Astrophys. J. Suppl. 18, 297 (1969).

${ }^{3}$ J. M. Ziman, Philos. Mag. 6, 1013 (1961).

${ }^{4}$ N. W. Ashcroft and J. Lekner, Phys. Rev. 145, 83 (1966).

${ }^{5}$ T. Wainwright and B. Alder, Nuovo Cimento Suppl. $\underline{9}$, 116 (1968)

${ }^{6}$ J. Hubbard, Proc. R. Soc. A 243,336 (1957).

${ }^{7} S$ is a function only of the combination $q \sigma$. Since $q=2 k_{F} y$, it follows from (7) that $q \sigma=2 y(18 \pi Z \eta)^{1 / 3}$ and the valence and packing fraction therefore enter in the combination $(Z \eta)^{1 / 3}$.

${ }^{8}$ D. Stroud and N. W. Ashcroft, Phys. Rev. B $\underline{5}, 371$ (1972).

"It is worth noting, however, that "softness" in the shortrange interaction does not substantially alter the form of $S(k)$ [see D. Schiff and J. P. Hansen, in Proceedings of the Second International Conference on the Properties of Liquid Metals (Taylor and Francis, London, 1973), p. 57].

${ }^{10}$ This follows from a simplistic application of the Wiedemann-Franz relation. The conductive opacity is proportional to the resistivity.

${ }^{11}$ N. W. Ashcroft and D. C. Langreth, Phys. Rev. 156, 685 (1967).

${ }^{12}$ Provided all spheres have the same diameters, we need not restrict ourselves to binary alloys.
${ }^{13}$ Following the methods of Ref. (8), the free energy of the alloy was minimized with respect to $\alpha$ and $\eta$, and the value of $\alpha$ obtained is consistent with a direct estimate from the form of the ion-ion interactions.

${ }^{14}$ W. B. Hubbard, Astrophys. J. 162, 687 (1970) (and other references given therein).

${ }^{15}$ V. P. Trubitsyn, Astron. Zh. $\underline{49}, 420$ (1972) [Sov. Astron.-AJ 16, 342 (1972)].

${ }^{16}$ Screening must be included [as in Eq. (4)]. For the unscreened interaction see, for example, J. E. Purcell, R. A. Berg, and A. E. S. Green, Phys. Rev. A ㅁ, 107 (1970).

${ }^{17}$ This estimate of the temperature results from the use of an adiabatic model in which we equate the surface entropy (Ref. 18) to that appropriate to the planetary center: The latter can be found by calculating the free energy of a hydrogen-helium liquid alloy using an extension of the method of Ref. (8).

${ }^{18}$ W. B. Hubbard, Astrophys. J. 152,745 (1968).

${ }^{19} \mathrm{R}$. Hide, in Magnetism and the Cosmos, edited by W. R. Hindmarsh, F. J. Lowes, P. H. Roberts and S. K. Runcorn (American Elsevier, New York, 1965), p. 378.

${ }^{20}$ H. H. Aumann, C. M. Gillespie, Jr., F. J. Lowes, Astrophys. J. 157, L69 (1969).

${ }^{21}$ D. Pines, Elementary Excitations in Solids (Benjamin, New York, 1963), p. 19.

${ }^{22}$ A. A. Abrikosov, Zh. Eksp. Teor. Fiz. 39, 1797 (1960) [Sov. Phys.-JETP 12, 1254 (1961)].

${ }^{23} \mathrm{~V}$. Trubitsyn, Fiz. Tverd. Tela $\underline{8}, 862$ (1966) [Sov. Phys.-Solid State 8, 688 (1966)].

${ }^{24}$ E. L. Pollock, J. $\vec{P}$. Hansen (unpublished); J. P. Hansen, Physics Lett. A 41, 213 (1972).

${ }^{25} \mathrm{G}$. Neece, F. Rogers, and W. Hoover, J. Comput. Phys. 7, 621 (1971). 